Research Article

\title{
Simulation of Motion of Long Flexible Fibers with Different Linear Densities in Jet Flow
}

\author{
Peifeng Lin (iD, ${ }^{1}$ Wenqian $\mathrm{Xu},{ }^{1}$ Yuzhen Jin, ${ }^{1}$ and Zefei Zhu $\mathbb{D}^{2}$ \\ ${ }^{1}$ Key Laboratory of Fluid Transmission Technology of Zhejiang Province, Zhejiang Sci-Tech University, Hangzhou 310008, China \\ ${ }^{2}$ School of Mechanical Engineering, Hangzhou Dianzi University, Hangzhou 310008, China \\ Correspondence should be addressed to Zefei Zhu; zzf.3691@163.com
}

Received 1 February 2018; Accepted 12 March 2018; Published 2 May 2018

Academic Editor: Mingzhou Yu

Copyright (c) 2018 Peifeng Lin et al. This is an open access article distributed under the Creative Commons Attribution License, which permits unrestricted use, distribution, and reproduction in any medium, provided the original work is properly cited.

Air-jet loom is a textile machine designed to drive the long fiber using a combination flow of high-pressure air from a main nozzle and a series of assistant nozzles. To make the suggestion of how to make the fiber fly with high efficiency and stability in the jet flow, in which vortices also have great influence on fiber movement, the large eddy simulation method was employed to obtain the transient flow field of turbulent jet, and a bead-rod chain fiber model was used to predict long flexible fiber motion. The fluctuation and velocity of fibers with different linear densities in jet flow were studied numerically. The results show that the fluctuation amplitude of a fiber with a linear density of $0.5 \times 10^{-5} \mathrm{~kg} \cdot \mathrm{m}^{-1}$ is two times larger than that of a fiber with a linear density of $2.0 \times 10^{-5} \mathrm{~kg} \cdot \mathrm{m}^{-1}$. The distance of the first assistant nozzle from the main nozzle should be less than $120 \mathrm{~mm}$ to avoid collision between the fiber and the loom. The efficient length of the main nozzle to carry the fiber flying steadily forward is about $100-110 \mathrm{~mm}$. For fibers with a linear density of $0.5 \times 10^{-5} \mathrm{~kg} \cdot \mathrm{m}^{-1}$, it is suggested that the distance of the first assistant nozzle from the main nozzle is about $110 \mathrm{~mm}$. With the increase of fiber linear density, the distance could be appropriately increased to $140 \mathrm{~mm}$. The simulation results provide an optimization option for the air-jet loom to improve the energy efficiency by reasonably arranging the first assistant nozzle.

\section{Introduction}

Carried flight of a long, flexible fiber in a jet flow field is a fundamental science problem for air-jet loom [1], which is designed to drive the yarn using a combination flow of highpressure air from a main nozzle and a series of assistant nozzles. Different from other pipeline pneumatic conveying systems, the jet flow of the main nozzle will rapidly decelerate in a free area and cannot guarantee a sustained highspeed flight of the fiber. Therefore, assistant nozzles must be added to the system. On the other hand, the presence of the reed groove requires that the fluctuation of the fiber must be less than a certain range. How to make the fiber fly forward rapidly and steadily is always a core issue of fiber flight and is jointly determined by two aspects: the fiber model and the flow simulation.

In general, a long fiber can be modeled as a rigid or flexible fiber for different dynamic research purposes. Yamamoto and
Matsuoka [2] proposed a method for simulating the dynamic behavior of rigid and flexible fibers in a flow field. The fiber is regarded as made up of spheres that are lined up and bound to each neighbor. Each pair of bonded spheres can stretch, bend, and twist by changing the bond distance, bond angle, and torsion angle between spheres, respectively. For the rigid fiber, the computed period of rotation and distribution of orientation angle agree with those calculated using Jeffery's equation with an equivalent ellipsoidal aspect ratio. For the flexible fiber, the period of rotation decreases rapidly with the growth of bending deformation of the fiber and rotation orbits deviate from a circular one for the rigid fiber. De Meulemeester et al. [3] tried to study the dynamic properties of fiber movement and developed a one-dimensional mathematical model, in which the behavior of the fiber is described by Newton's second law. Lindström and Uesaka [4] proposed a model for flexible fibers in viscous fluid flow; namely, the fibers are modeled as chains of fiber segments which interact 
with the fluid through viscous and dynamic drag forces. Fiber segments, from the same or different fibers, interact with each other through normal, frictional, and lubrication forces. The simulations using the proposed model successfully reproduced the different regimes of motion for thread-like particles that range from rigid fiber motion to complicated orbiting behavior including coiling and self-entanglement.

Vahidkhah and Abdollahi [5] used the lattice Boltzmann method (LBM) to solve the Newtonian flow field and the immersed boundary method (IBM) to simulate the deformation of the flexible fiber interacting with the flow. The variations of the fiber length during the simulation time for different values of stretching constant are studied. Kabanemi and Hétu [6] carried out a direct simulation study to analyze the effect of fiber rigidity on fiber motion in simple shear flow. The fiber is modeled as a series of rigid spheres connected by stiff springs, which is similar to that used by Yamamoto and Matsuoka [2]. The model correctly predicts the orbit period of fiber rotation, as well as the trend of critical flow strength, versus fiber aspect ratio, during which the breakage occurs in simple shear flow. Meirson and Hrymak [7] extracted rotational friction coefficients from Jeffery's model, created a general case long flexible fiber orientation model, and applied it in a simple shear flow. Nan et al. [8] presented a linear viscoelastic sphere-chain model based on the discrete element method to quantify the material damping of deformed flexible fibers. A correlation is formulated to quantify the relationship between the damping coefficient of the local bond and that of the flexible fiber. Meulemeester et al. [9] developed a threedimensional mathematical model of the yarn. The threedimensional model for the weft insertion on air-jet looms has been successfully tested.

On the other side, as we have mentioned, fiber flying in fluid is also affected by turbulent flow characteristics. Kim et al. [10] analyzed the flow in an air-jet loom by using a time-accurate characteristic-based upwind flux-difference splitting compressible Navier-Stokes method. The unsteady pressure and Mach number behavior along the center line of the main nozzle were analyzed. Andrić et al. [11] analyzed the dynamics of individual flexible fibers in a turbulent flow, the direct numerical simulation of the incompressible NavierStokes equations is used to describe the fluid flow in a plane channel, and a one-way coupling is considered between the fibers and the fluid phase. They found that the fiber motion is primarily governed by velocity correlations of the flow fluctuations. In addition, they reported that there is a clear tendency of the thread-like fibers to evolve into complex geometrical configurations in a turbulent flow field, and the fiber inertia has a significant impact on reorientation timescales of fibers suspended in a turbulent flow field. Jin et al. [12] simulated the turbulent flow by solving the Reynoldsaveraged Navier-Stokes (RANS) equations and conducted the three-dimensional numerical simulation of the movement of the flexible body. The numerical results show that an unconstrained flexible body would turn over forward along the airflow's diffusion direction, while a constrained flexible body in the flow field will make a periodic rotation motion along the axis of the flexible

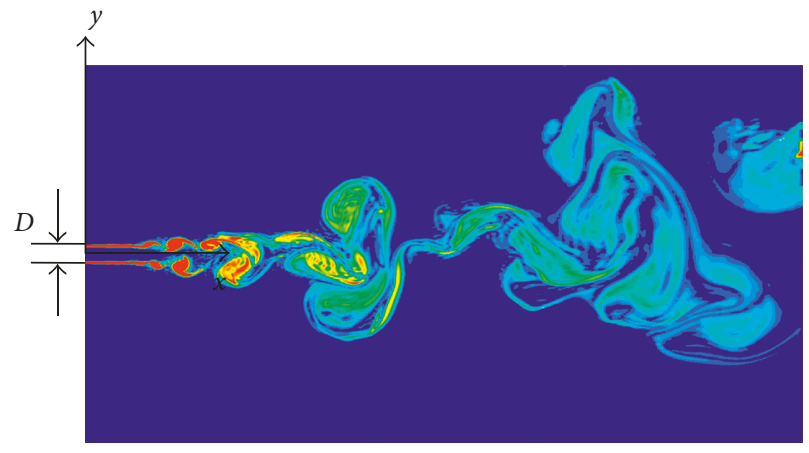

FIGURE 1: Schematic diagram of vortices in jet flow.

body, and the bending deformation is more obvious than that of unconstrained flexible body. Yang et al. [13] studied the two-way coupling turbulent model and rheological properties for fiber suspension in the contraction based on the RANS simulation.

Pei and Yu [14] studied the motional characteristics of the flexible fibers in the airflow inside the Murata vortex spinning (MVS) nozzle. A two-dimensional fluid structure interaction (FSI) model combined with the fiber wall contact is introduced to simulate a single fiber moving in the airflow inside the MVS nozzle. The model is solved using a finite element code ADINA. Based on their simulation results, the formation principle and the influence of some nozzle parameters on the tensile property of the MVS fiber were discussed.

More and more investigators put their interest on the air-jet loom [15-18] and other fluid machineries [19, 20], but due to the computation cost gap between scientific researches and engineering needs, only a few of them considered the influence of turbulent fluctuation [21]. The jet flow caused by the main nozzle is a typical free shear turbulence, and there are strong vortices which play an important role on the momentum and energy transport of flow field $[22,23]$. The vortices also have great influence on the fiber movement. Large eddy simulation (LES) is a believed mathematical model for turbulence vortex simulation, by directly calculating the large-scale flow motion.

In this paper, we simplified the two-phase flow system and were able to employ the LES method to simulate the development of a vortex in jet flow and the Lagrangian beadrod model to give the time evolution of a long flexible fiber distribution with different linear densities. Then, the fiber fluctuation and the velocity were discussed to make the suggestion of how to make the fiber fly with high efficiency and stability in an air-jet loom.

\section{Mathematical Formulation}

2.1. Fluid Flow. The 2D jet flow is shown in Figure 1, in which $x$ and $y$ are the streamwise and cross-stream directions, respectively. The width of the nozzle $D$ is $3.5 \mathrm{~mm}$, the fluid velocity at the nozzle $U_{0}$ is $240 \mathrm{~m} / \mathrm{s}$, and the flow Reynolds number $\operatorname{Re}=5.68 \times 10^{4}$. 
The LES equations governing the jet flow obtained by filtering the Navier-Stokes equations are as follows [24]:

$$
\begin{gathered}
\frac{\partial \rho}{\partial t}+\frac{\partial\left(\rho \mathbf{u}_{n}\right)}{\partial x_{n}}=0 \\
\frac{\partial\left(\rho \mathbf{u}_{m}\right)}{\partial t}+\frac{\partial\left(\rho \mathbf{u}_{m} \mathbf{u}_{n}\right)}{\partial x_{n}}=\frac{-\partial \mathbf{p}}{\partial x_{m}}+\frac{\partial}{\partial x_{n}}\left(\mu \frac{\partial \sigma_{m n}}{\partial x_{n}}-\tau_{m n}\right),
\end{gathered}
$$

where $\rho$ is the fluid density, $\mathbf{u}_{m}$ is the filtered velocity, $\mathbf{p}$ is the filtered pressure, index $m, n$ is taken as 1,2 and refers to the $x, y$, and $\mu$ is the dynamic viscosity.

The SGS (subgrid stress) tensor $\tau_{m n}$ and SmargorinskyLilly model [24] which are based on the mixing length hypothesis are used to calculate the SGS stress.

2.2. Flexible Fiber Model. A single flexible long fiber is modeled as a bead-rod chain, which is similar to that used by Guo et al. [25]. The fiber model is composed of $N$ beads, which are connected by $N-1$ massless rods (Figure 2). Only the beads are affected by forces, and the rods maintain the configuration of the fiber. Using the model, the chain is allowed to be stretched by changing the distance of adjacent rods.

If the distance between adjacent beads is not equal to the equilibrium distance, the stretching restoring force $\mathbf{F}_{\mathrm{n} i}$ exerted on the bead $i$ will be

$$
\mathbf{F}_{\mathrm{n} i}=-\frac{\pi R^{2} E}{L} \Delta \mathbf{L}
$$

where $R$ is the bead radius, $E$ is the elastic modulus, $L$ is the equilibrium distance, and $\Delta \mathbf{L}$ is the distance variation, which is equal to the transient distance of each two adjacent beads subtracted by the equilibrium distance.

When immersed in the unsteady flow field which is calculated in the last section, the fiber is subjected to hydrodynamic forces, which are also changed with time. In this paper, only drag force is considered in order to decrease the computational cost. Other hydrodynamic forces such as Basset history term, additional mass, slip-rotational lift force, and fluid inertia are negligible. For bead $i$, the drag force $\mathbf{F}_{\mathrm{d} i}$ is contributed by fiber sections $(i-1, i)$ and $(i, i+1)$. It can be calculated as follows:

$$
\mathbf{F}_{\mathrm{d} i}=\frac{1}{2}\left(\mathbf{F}_{\mathrm{d} i-1, i}^{i}+\mathbf{F}_{\mathrm{d} i, i+1}^{i}\right),
$$

where $\mathbf{F}_{\mathrm{d} i-1, i}$ and $\mathbf{F}_{\mathrm{d} i, i+1}$ are the drag forces acting on bead $i$, which are devoted to the fiber section $(i-1, i)$ and $(i, i+1)$, respectively. The drag $\mathbf{F}_{\mathrm{d} i-1, i}$ acting on the fiber section $(i-1, i)$ can be expressed as follows:

$$
\mathbf{F}_{\mathrm{d} i-1, i}^{i}=\frac{\pi r_{v}^{2}}{2} C_{\mathrm{d}} \rho\left|\mathbf{V}_{\mathrm{q} i}-\mathbf{V}_{\mathrm{d} i}\right|\left(\mathbf{V}_{\mathrm{q} i}-\mathbf{V}_{\mathrm{d} i}\right)
$$

where $\mathbf{V}_{\mathrm{q} i}$ and $\mathbf{V}_{\mathrm{d} i}$ are the fluid and fiber velocities at the mass center of the fiber section $(i-1, i)$, and $C_{\mathrm{d}}$ is the drag coefficient associated with Reynolds number, which can be represented as follows:

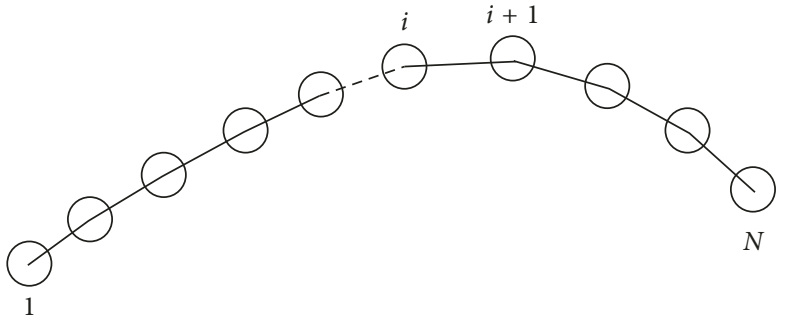

Figure 2: Schematic diagram of the flexible fiber model.

$$
C_{d}= \begin{cases}\frac{24}{\operatorname{Re}} & \operatorname{Re} \leq 1 \\ \frac{24}{\operatorname{Re}}+\frac{4}{\sqrt[3]{\operatorname{Re}}} & 1<\operatorname{Re} \leq 1000 \\ \beta & \operatorname{Re}>1000\end{cases}
$$

where $\operatorname{Re}_{s}=2 r_{v} \rho\left|\mathbf{V}_{\mathrm{q} i}-\mathbf{V}_{0 i}\right|$ is the Reynolds number of equivalent spherical and $\beta$ is a constant between 0.4 and 0.45 . According to Newton's second law, the equations of motion for the bead $i$ that constitute the fiber are as follows:

$$
\begin{aligned}
m_{i} \frac{d^{2} \mathbf{r}_{i}}{d t^{2}} & =\mathbf{F}, \\
\mathbf{F} & =\mathbf{F}_{\mathrm{n} i}+\mathbf{F}_{\mathrm{d} i},
\end{aligned}
$$

where $m_{i}$ is the mass of the bead $i$ and $\mathbf{r}_{i}$ is the position vector of the bead $i$.

\section{Results and Discussion}

3.1. Computation Conditions. The computational domain covers $x \times y=21 D \times 40 D$, the nozzle width $D=3.5 \mathrm{~mm}$, fully developed boundary conditions at the outlet, and static surrounding environment conditions are assumed. The local mesh is shown in Figure 3. Fiber motion is solved in one-way coupling between the fiber and flow; fiber-wall and fiberfiber interactions are neglected.

\subsection{Method Verification}

3.2.1. Jet Flow Field Verification. According to the experimental results of $[26,27]$, the streamwise velocity satisfies the self-preservation distribution at $x \geq 8 D$ and the profile is expressed as a Gaussian curve. The self-preservation profiles of the mean velocity are shown in Figure 4, where the data are normalized by the centerline mean velocity $U_{m}$ and the half-width $r_{0.5}$, which is the distance between the position where the streamwise velocity $u / U_{\mathrm{m}}=0.5$ and the center line of the jet. The figure shows that the air flow which is mentioned in Section 2.1 has been successfully simulated.

3.2.2. Flexible Fiber Model Verification. As the limitation of the visible area and the resolution of the high-speed camera, an experiment about the motion of microscale fixed flexible 


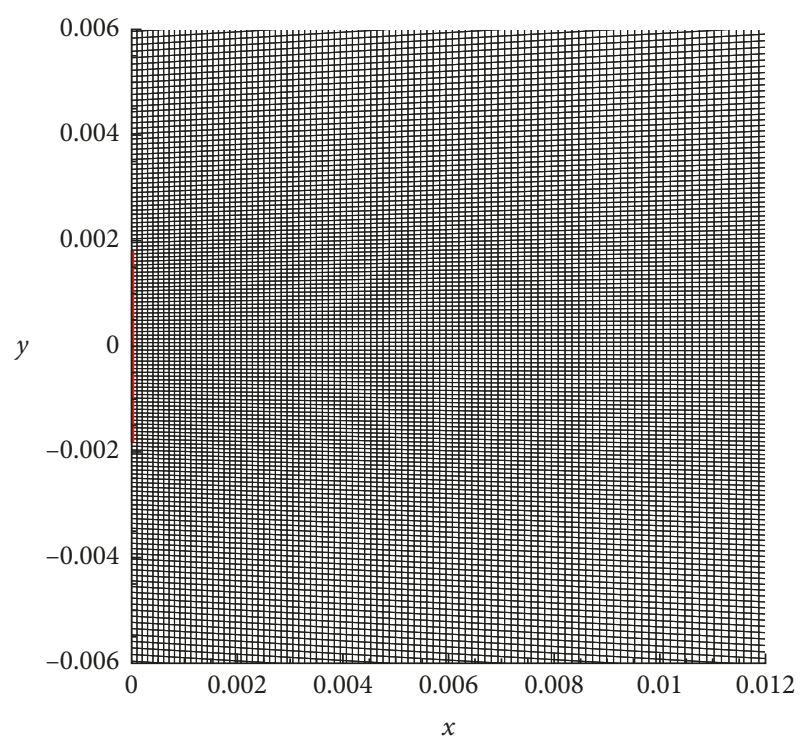

Figure 3: Mesh at the nozzle exit.

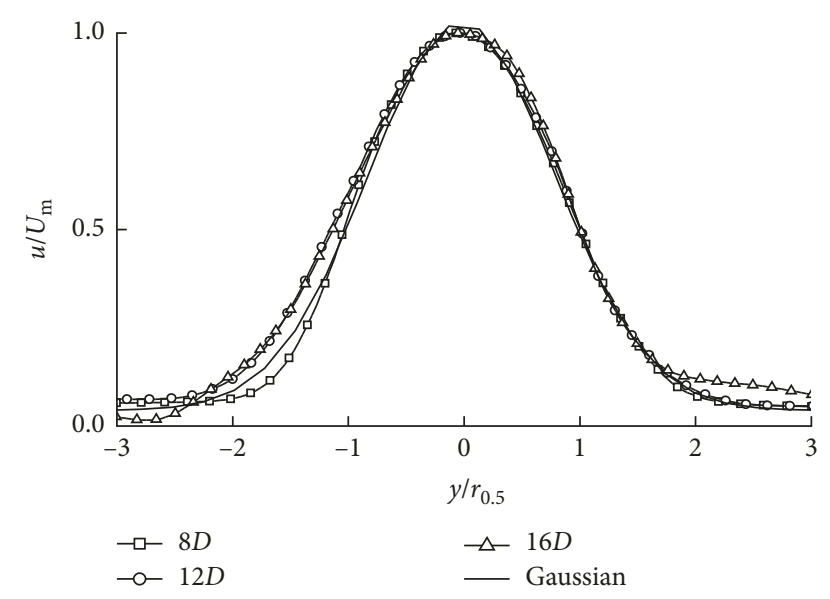

FIGURE 4: Self-preservation profiles of the mean streamwise velocity by the LES method.

fiber was carried out to verify the long flexible fiber model. The fixed fiber length $L_{\mathrm{f}}=100 \mathrm{~mm}$, and the linear density (mass per unit length) $\rho_{\mathrm{L}}=0.2 \times 10^{-5} \mathrm{~kg} \cdot \mathrm{m}^{-1}$. As shown in Figure 5, the noncontact test bed consists of an air jet loom, high-speed camera, and professional image analysis software Image Pro-Plus. The fiber is fixed at the nozzle so its motion state can be recorded by the high-speed camera after each parameter adjustment of the loom.

The transient fiber shape comparison of numerical simulation results and that recorded by the high-speed camera are shown in Figure 6. It can be seen that the It can be seen that the free end of the fiber fluctuates significantly all the time, but the fluctuation of the fiber segment close to the main nozzle is not obvious. fluctuates significantly all the time, but the fluctuation of the fiber segment close to the main nozzle is not obvious. This is due to the turbulence characteristics of the jet flow, fiber flexibility, and the shape characteristics of the fiber.

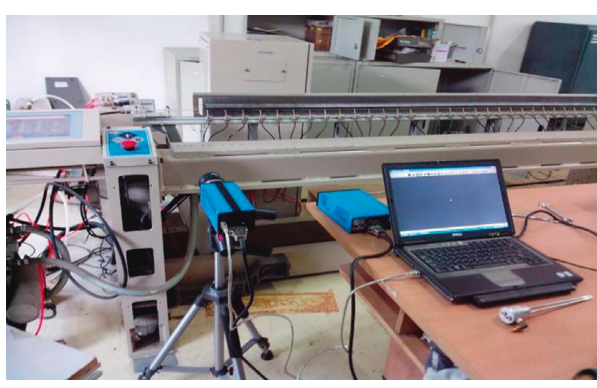

Figure 5: Test equipment of the experiment.

There are some less significant differences between the simulation models used in this paper and the actual fiber properties: the bending modulus of the fiber, which is much lower than the elasticity modulus, is not taken into account; the hydrodynamic forces such as Basset force and sliprotational lift force, which are relative small compared to the drag force, are neglected as well. Despite all the simplifications, the experimental and simulation results are still in relatively good agreement (Figure 6). Therefore, the following simulation of the flight fiber is reasonable and credible.

3.3. Discussions and Analysis. When a micro- or nanoscale free fiber is being carried by the high-velocity airflow after being injected into the jet flow field, as described above, the flexible fiber model assumes that the fiber mass is concentrated at a series of beads, and the intervals between every two beads can vary as a result of the force-displacement balance on the beads. Subsequently, the transient equivalent radius of the fiber varies too; they can even be reduced from microscale to nanoscale. Fiber linear density is another important characteristic and has a significant effect on calculating the force acted on the beads which would determine the fiber motion in the jet flow. So the fiber motion with different linear densities of $\rho_{\mathrm{L}}=0.5 \times 10^{-5}, 1.0 \times 10^{-5}$, $1.5 \times 10^{-5}$, and $2.0 \times 10^{-5} \mathrm{~kg} \cdot \mathrm{m}^{-1}$ are separately studied.

The initial velocity of the fiber is $v_{\text {in }}=70 \mathrm{~m} / \mathrm{s}$. The initial length of static free fiber is $L_{\mathrm{f}}=40 \mathrm{~mm}$. The initial bead radius is given as $R=100 \mu \mathrm{m}$. The two ends of the fibers are marked as end A and B, respectively. At the initial stage, end $A$ is at the position $x=40 \mathrm{~mm}$ and end $B$ is at $x=0 \mathrm{~mm}$ (Figure 7). The motional characteristic of the fiber is stable at the initial stage. The farther from the nozzle, the lower the air velocity. So, the velocity of end $B$ will be faster than that of end A after a period of time. As a result, fiber bending deformation is observed.

3.3.1. Effect of Fiber Linear Density on Fiber Fluctuation. Due to different densities, the time of fiber flying across the simulation domain is different. The fiber movement is analyzed until the end $\mathrm{A}$ of fiber arrives at the calculating boundary.

The motion and bending deformation of fibers with different linear densities over time are shown in Figure 8. As the jet flow goes on, the vortex keeps rolling-up, transporting 


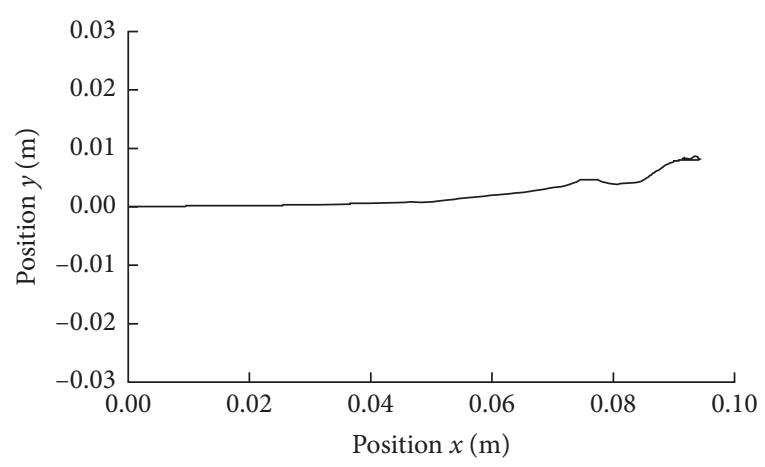

$-0.0030 \mathrm{~s}$

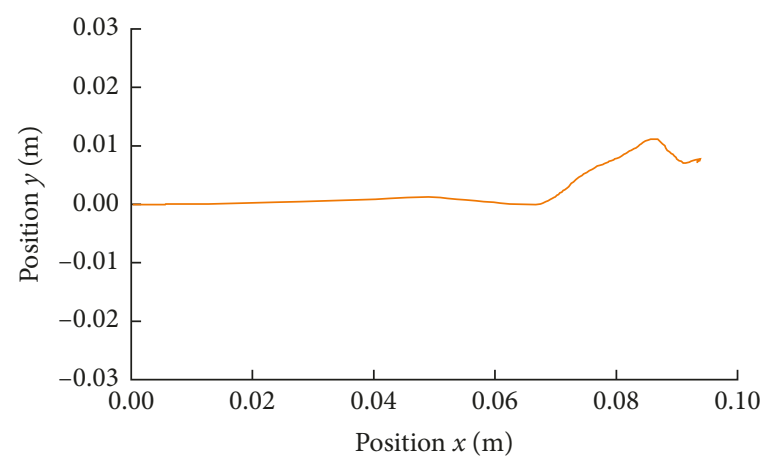

$-0.0040 \mathrm{~s}$

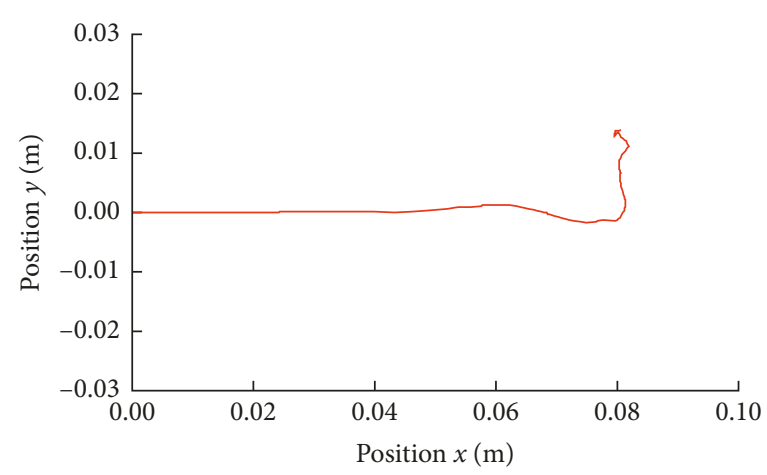

$-0.0050 \mathrm{~s}$

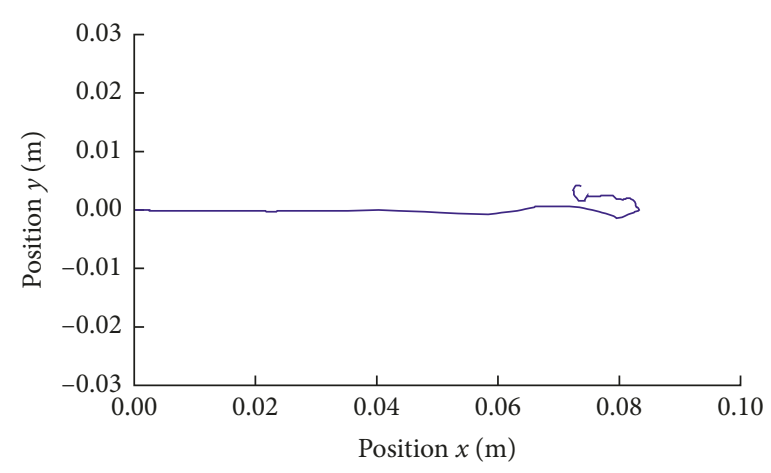

$-0.0055 \mathrm{~s}$

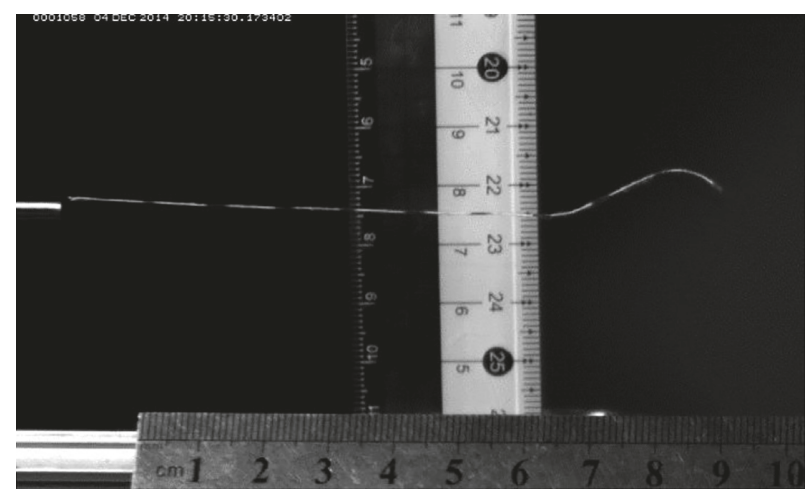

(a)

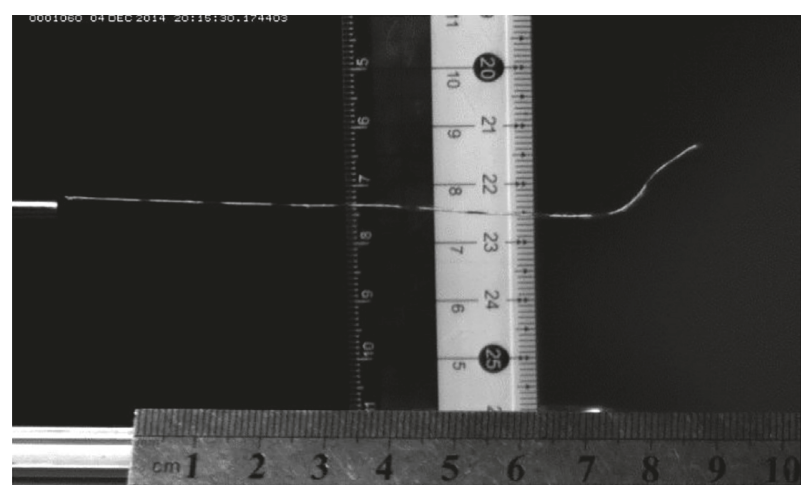

(b)

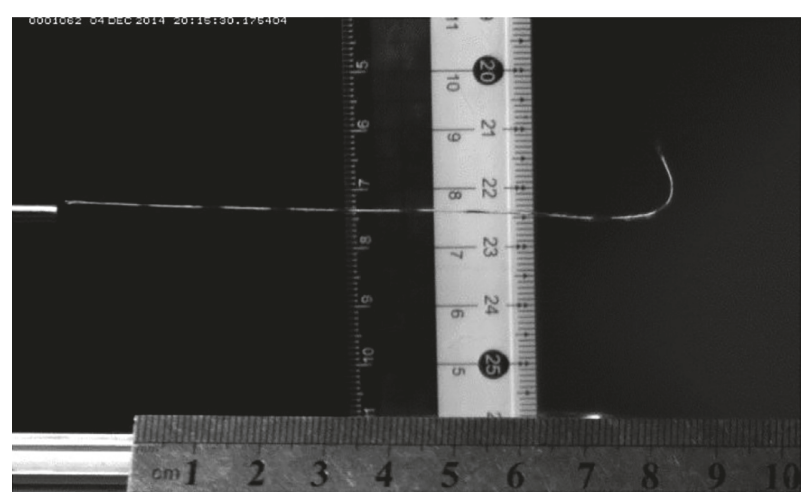

(c)

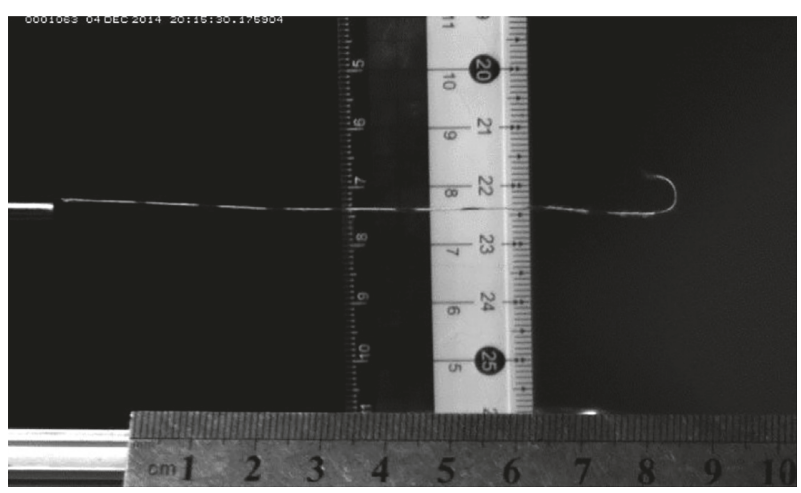

(d)

Figure 6: Result comparison of fiber between numerical simulation and high-speed photography. 


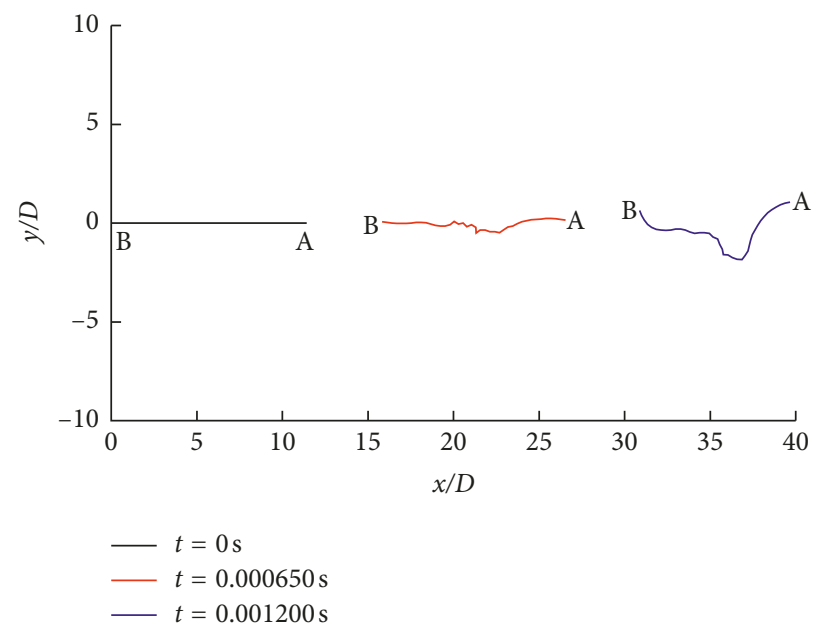

Figure 7: Motion of free fiber in jet flow.

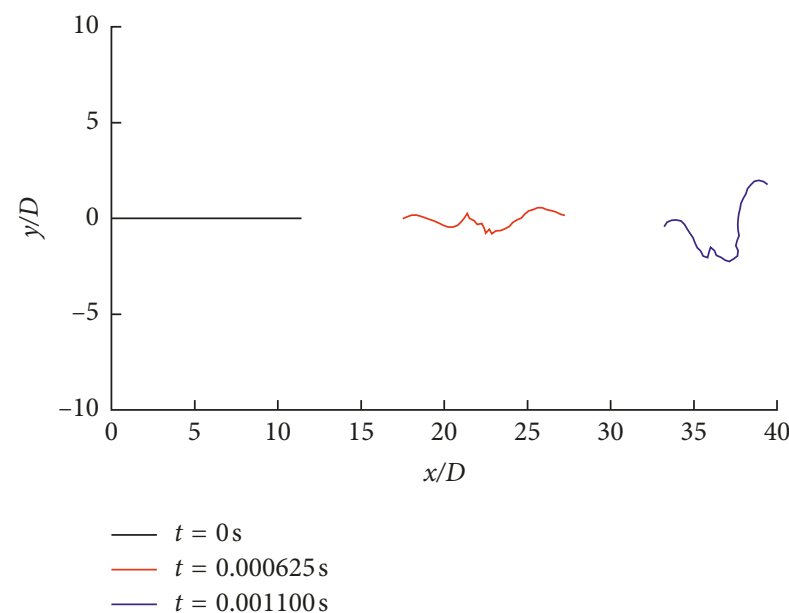

(a)

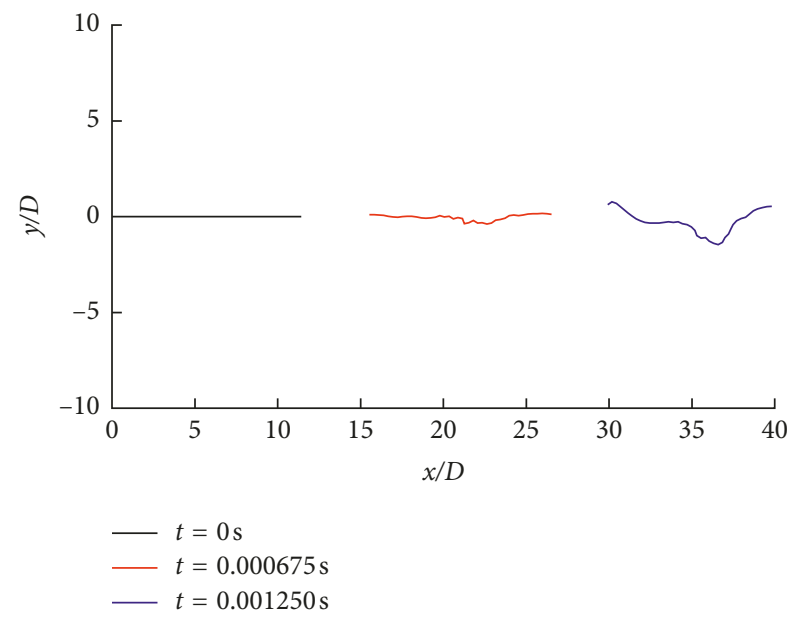

(c)

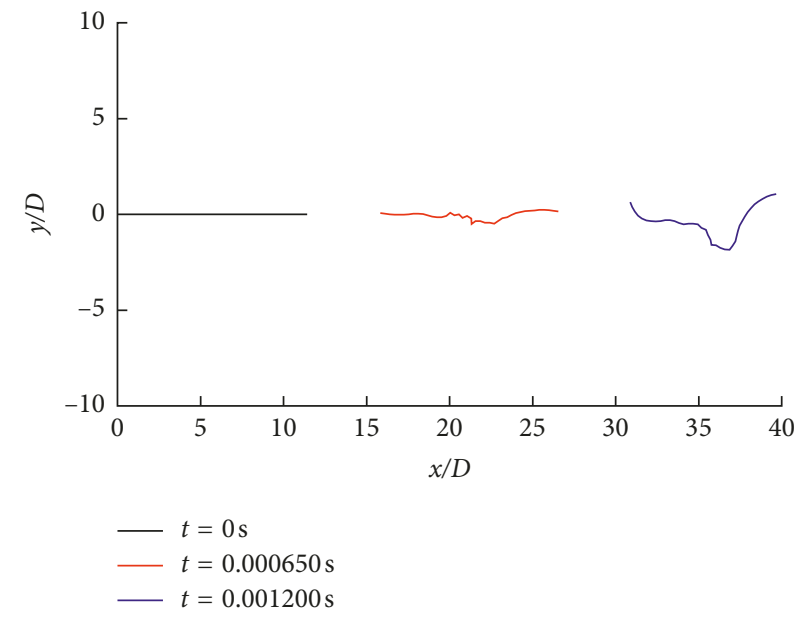

(b)

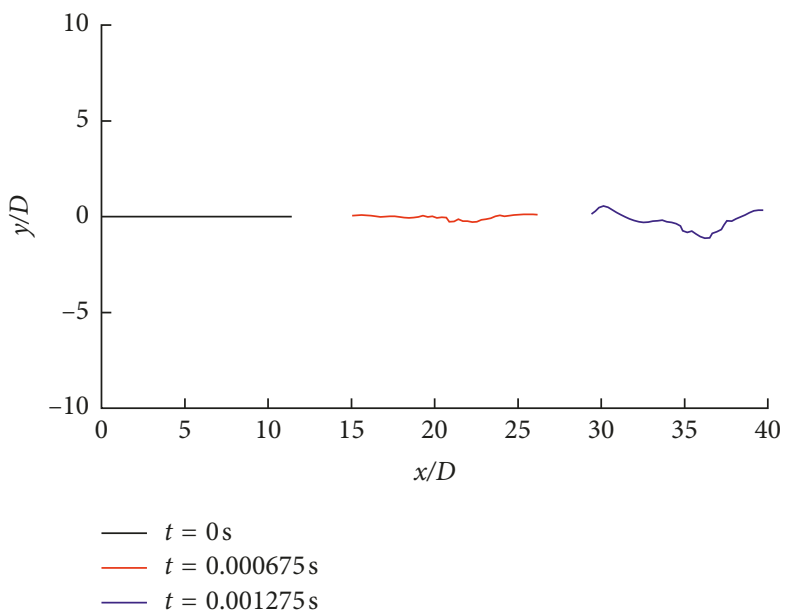

(d)

Figure 8: Motional characteristic of the fiber with different linear densities: (a) $\rho_{\mathrm{L}}=0.5 \times 10^{-5} \mathrm{~kg} \cdot \mathrm{m}^{-1},(\mathrm{~b}) \rho_{\mathrm{L}}=1.0 \times 10^{-5} \mathrm{~kg} \cdot \mathrm{m}^{-1}$, (c) $\rho_{\mathrm{L}}=1.5 \times 10^{-5} \mathrm{~kg} \cdot \mathrm{m}^{-1}$, and (d) $\rho_{\mathrm{L}}=2.0 \times 10^{-5} \mathrm{~kg} \cdot \mathrm{m}^{-1}$. 
and mixing with each other (Figure 1), and the fluctuation of vortex driven on fiber becomes more and more strong. By comparing Figures $8(\mathrm{a})-8(\mathrm{~d})$, it can be seen that the fiber fluctuation relating with linear density $\rho_{\mathrm{L}}=0.5 \times 10^{-5} \mathrm{~kg} \cdot \mathrm{m}^{-1}$ is more obvious than that of fiber with linear density $\rho_{\mathrm{L}}=2.0 \times 10^{-5} \mathrm{~kg} \cdot \mathrm{m}^{-1}$, which shows that the smaller linear density causes the more unstable fiber motion. This is because the fiber transverse acceleration generates from the velocity difference between transverse velocity of flow field and that of fiber. Moreover, the smaller linear density results in the larger transverse acceleration and the larger transverse acceleration lead to the more unstable fiber motion.

The transverse velocity of end A of fibers with different linear densities is shown in Figure 9. The fiber transverse velocity is $0 \mathrm{~m} / \mathrm{s}$ at the initial time, and it becomes larger with the increasing of running time. The end transverse velocity of fiber with linear density $\rho_{\mathrm{L}}=2.0 \times 10^{-5} \mathrm{~kg} \cdot \mathrm{m}^{-1}$ increases to about $2 \mathrm{~m} / \mathrm{s}$ after $0.0012 \mathrm{~s}$. At the same time, the end transverse velocity of fiber with linear density $\rho_{\mathrm{L}}=0.5 \times$ $10^{-5} \mathrm{~kg} \cdot \mathrm{m}^{-1}$ increases to about $18 \mathrm{~m} / \mathrm{s}$. Thus, the smaller fiber linear density leads to the larger transverse velocity. In other words, the fiber transverse fluctuation becomes more obvious.

In order to learn more about the influence of air velocity on fiber fluctuations, several equidistant points are selected on the fiber (Figure 10). To represent the amplitude of fiber fluctuation, the fluctuation standard deviation of fiber is calculated using (7) when the end A of fiber arrives at $40 \mathrm{~mm}$, $80 \mathrm{~mm}, 100 \mathrm{~mm}, 120 \mathrm{~mm}$, and $140 \mathrm{~mm}$ (i.e., $x_{\mathrm{A}}=40 \mathrm{~mm}$, $80 \mathrm{~mm}, 100 \mathrm{~mm}, 120 \mathrm{~mm}$, and $140 \mathrm{~mm}$ ) from the main nozzle:

$$
\sigma=\sqrt{\frac{\sum \Delta y^{2}}{n}}
$$

where $\Delta y$ is the vertical distance from the point to the center line and $n$ is the sum of the selected points in the fiber $(n=41)$. The fluctuation standard deviation is listed in Table 1.

As shown in Table 1, the fiber is affected by the airflow and the fiber fluctuation standard deviation increases gradually in the motion process with time. When the fiber with different linear densities arrives at the same location in jet flow, the smaller fiber linear density causes the greater fluctuation standard deviation. The fluctuation amplitude of fibers with $\rho_{\mathrm{L}}=0.5 \times 10^{-5} \mathrm{~kg} \cdot \mathrm{m}^{-1}$ is two times larger than that of fibers with $\rho_{\mathrm{L}}=2.0 \times 10^{-5} \mathrm{~kg} \cdot \mathrm{m}^{-1}$. That is to say, the fiber with smaller linear density will fluctuate more obviously as it flies across the computation domain. As the cross section of the reed groove in the loom is about $5 \mathrm{~mm} \times 5 \mathrm{~mm}$, so when the fluctuating amplitude of the fiber end is larger than $2.5 \mathrm{~mm}$, that is, after the head of fiber with $\rho_{\mathrm{L}}=0.5 \times 10^{-5} \mathrm{~kg} \cdot \mathrm{m}^{-1}$ arrived at $x_{\mathrm{A}}=120 \mathrm{~mm}$ and the head of fiber with $\rho_{\mathrm{L}}=1.0 \times 10^{-5} \mathrm{~kg} \cdot \mathrm{m}^{-1}$ arrived at $x_{\mathrm{A}}=140 \mathrm{~mm}$, the fiber will much more likely impact with the groove if there is no another assistant jet flow. So it is strongly recommended that the distance of the first assistant nozzle from the main nozzle is less than $120 \mathrm{~mm}$ for the fiber whose linear density is less than $0.5 \times 10^{-5} \mathrm{~kg} \cdot \mathrm{m}^{-1}$.

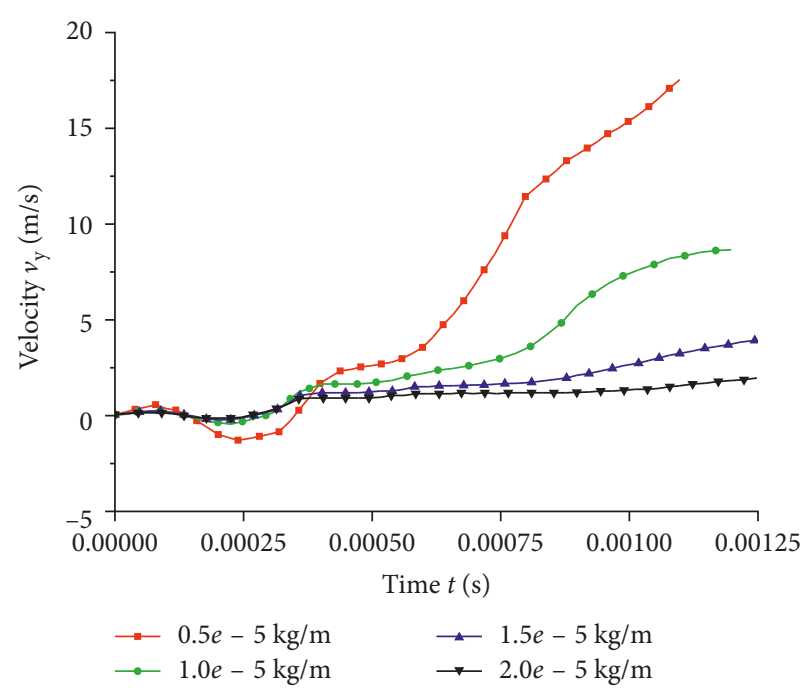

Figure 9: Transverse velocity distribution of end A of fibers with different linear densities.

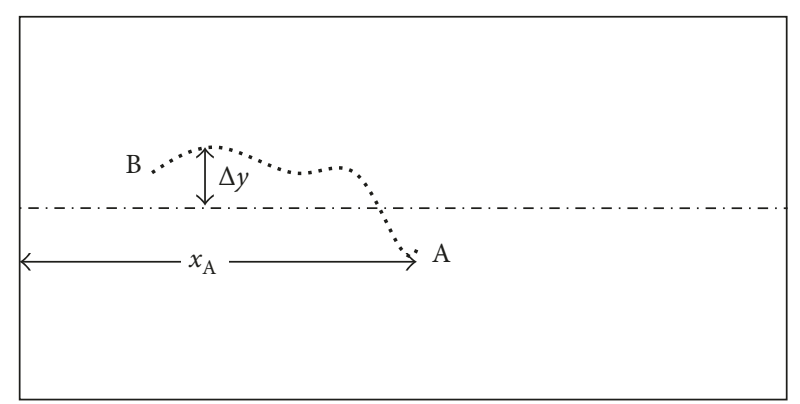

FIGURE 10: Illustration of free fiber in jet flow.

With the increase of fiber linear density, the distance could be appropriately increased.

3.3.2. Effect of Fiber Linear Density on Fiber Velocity. The $x$-velocity $\left(v_{\mathrm{A}}\right)$ and distance from the nozzle $\left(x_{\mathrm{A}}\right)$ of end A with different linear densities and time are shown in Figure 11. The corresponding flow axial velocity (at the positions where the end $\mathrm{A}$ is) is given in Figure 12. Within the running time of $0-0.0002 \mathrm{~s}$, the fiber velocity increases obviously. Then, the fiber is in a state of slow acceleration during the time of $0.0002-0.0004 \mathrm{~s}$. At the next stage, there is no longer a significant change on fiber velocity. After about $0.0008 \mathrm{~s}$, the fiber is in a state of deceleration. It can be explained as follows. The fiber velocity is relatively low at the initial moment. When the end A velocity is around $70 \mathrm{~m} / \mathrm{s}$, the air velocity of flow field is around $160 \mathrm{~m} / \mathrm{s}$ (Figure 12). There is a large velocity difference between the flow field and fiber. The fiber is in rapid acceleration because of the large drag force as listed previously. It means the fiber gets a lot of energy from the flow in the period of $0-0.0002 \mathrm{~s}$, and the end A flies about $15 \mathrm{~mm}$ forward in this ultra-short time. Then, the velocity of flow field, where the fiber is, decreases rapidly, which we can see from Figure 12. During the time of 
TABle 1: Standard deviation of fiber fluctuations with different fiber linear densities at different positions.

\begin{tabular}{lccccc}
\hline $\begin{array}{l}\text { Linear density } \\
\rho_{\mathrm{L}}\left(\mathrm{kg} \cdot \mathrm{m}^{-1}\right)\end{array}$ & \multicolumn{4}{c}{ Amplitude of fiber fluctuation, $\sigma(\mathrm{mm})$} \\
\hline $0.5 \times 10^{-5}$ & $x_{\mathrm{A}}=40 \mathrm{~mm}$ & $x_{\mathrm{A}}=80 \mathrm{~mm}$ & $x_{\mathrm{A}}=100 \mathrm{~mm}$ & $x_{\mathrm{A}}=120 \mathrm{~mm}$ & 3.34 \\
$1.0 \times 10^{-5}$ & 0 & 0.75 & 1.62 & 2.04 & $x_{\mathrm{A}}=140 \mathrm{~mm}$ \\
$1.5 \times 10^{-5}$ & 0 & 0.40 & 1.01 & 1.54 & 3.03 \\
$2.0 \times 10^{-5}$ & 0 & 0.29 & 0.75 & 1.13 \\
\hline
\end{tabular}

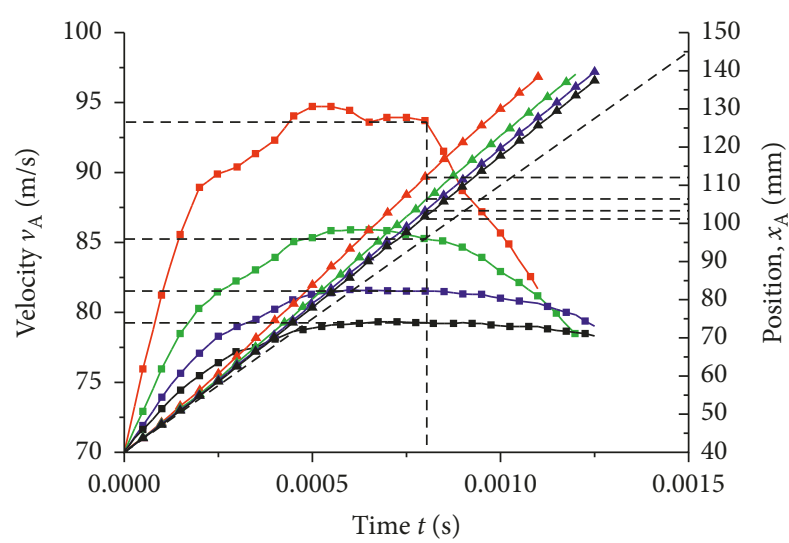

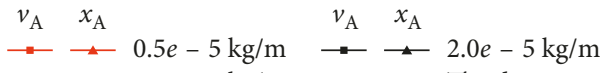

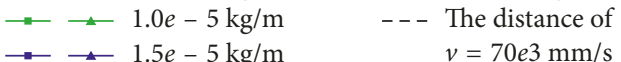

FIGURE 11: $v_{\mathrm{A}}$ and $x_{\mathrm{A}}$ curves with different linear densities and time.

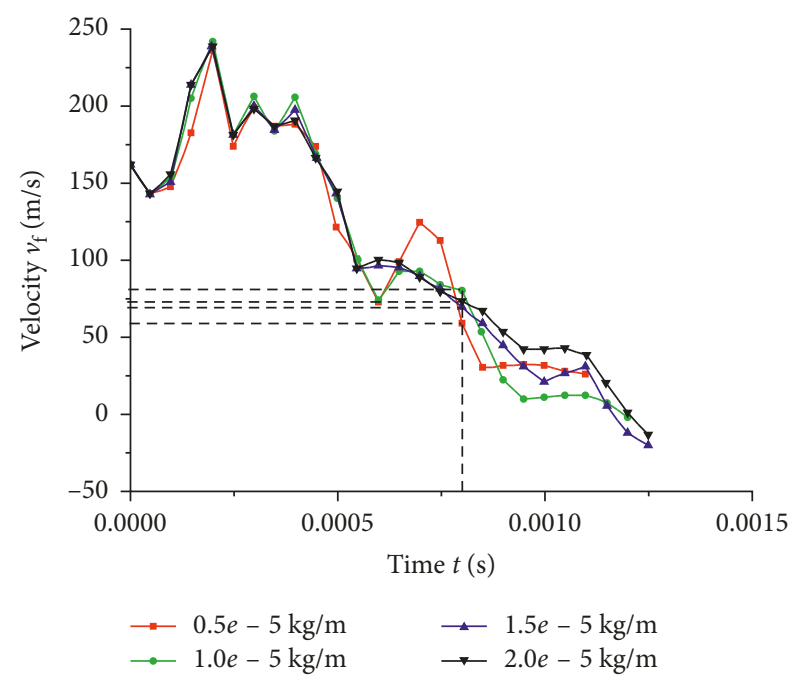

Figure 12: Airflow velocity at the position of fiber end A.

$0.0002-0.0005 \mathrm{~s}$, the velocity of airflow is larger than the fiber, but the velocity difference decreases. So the fiber is in a state of slow acceleration. As the velocity difference between the flow field and fiber keeps decreasing in 0.0005-0.0008 s, the influence of flow field on fiber velocity becomes unapparent and the fiber remains at the same velocity magnitude especially for the fibers with a relatively large linear density. At $t=0.0008 \mathrm{~s}$, the fiber end A arrives around $100 \mathrm{~mm}$ away from the main nozzle, which we can see from

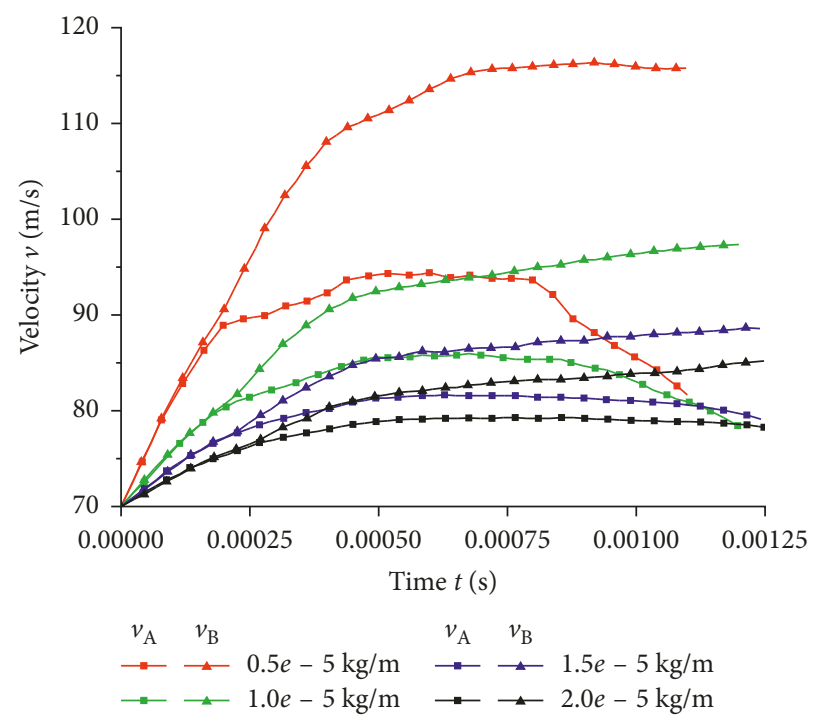

Figure 13: $X$-velocity of ends A and B of fiber with different linear densities.

Figure 11, and the velocity of airflow is lower than that of the fiber. The fiber cannot get energy from the flow any more for all the fibers simulated here. In other words, the efficient length of the main nozzle to carry the fiber flying rapidly forward is about $100-110 \mathrm{~mm}$. So the addition of an assistant nozzle is suggested in this place.

Besides, the difference of velocity distribution is obvious with different fiber linear densities. According to Newton's second law, the lower linear density leads to greater fiber acceleration. So when the velocity of airflow is greater than that of the fiber, the greater fiber acceleration causes the greater velocity. But, when the velocity of airflow is lower than that of the fiber, the velocity of fiber with a lower density falls quickly. It means that the fiber with lower linear density is more sensitive to the flow, and the flow velocity change along the fiber will easily cause a nonuniform velocity distribution at different segments of the fiber.

The $x$-velocity of ends A and B of the fiber with different linear densities is shown in Figure 13. The velocity difference between end $\mathrm{A}$ and $\mathrm{B}$ of the fiber with liner density $\rho_{\mathrm{L}}=$ $0.5 \times 10^{-5} \mathrm{~kg} \cdot \mathrm{m}^{-1}$ is about $35 \mathrm{~m} / \mathrm{s}$ when the end $A$ arrives at the calculating boundary, and the fiber with linear density $\rho_{\mathrm{L}}=2.0 \times 10^{-5} \mathrm{~kg} \cdot \mathrm{m}^{-1}$ is only about $10 \mathrm{~m} / \mathrm{s}$. The nonuniform velocity distribution leads to obvious bending deformation of the fiber (Figure $8(\mathrm{a})$ ), which is undesirable for pneumatic conveying especially jet loom. In order to guarantee the stability of movement of the fiber with lower 
density, a more stable velocity distribution of flow is needed. On the other hand, the decrease of the distance between first the assistant nozzle and the main nozzle can reduce the axial velocity change and improve the stability of flow [28]. So, in order to make the flight more stable, for the fiber with lower linear density, the distance of first assistant nozzle from the main nozzle should be appropriately smaller. But, it is obviously worse for saving energy. Actually, in industrial production, people will try their best to add the spacing to make full use of the high-speed air jet flow.

The discussions above are summarized as follows. First, as we have discussed before, for fiber with linear density $\rho_{\mathrm{L}}=0.5 \times 10^{-5} \mathrm{~kg} \cdot \mathrm{m}^{-1}$, if there is an assistant nozzle within $120 \mathrm{~mm}$ from the main nozzle, it will be highly likely for the fiber to impact with the groove. Second, the efficient length of the main nozzle to carry the fiber forward is less than $110 \mathrm{~mm}$. Third, to make full use of the high-speed air jet flow, the distance between the main nozzle and the first assistant nozzle cannot be very small, although the smaller nozzle spacing could lead to a more stable fiber flight. Considering all the factors, there is only a narrow range of suggestion distance to set the first assistant nozzle. For fiber with a linear density $\rho_{\mathrm{L}}=0.5 \times 10^{-5} \mathrm{~kg} \cdot \mathrm{m}^{-1}$, it is $110 \mathrm{~mm}$, and when the fiber linear density increases, the distance could be appropriately increased to $140 \mathrm{~mm}$.

\section{Conclusions}

To make the suggestion of how to make the fiber fly with high efficiency and stability in a jet flow, we employed the LES method to simulate the development of vortices in the jet flow and Lagrangian bead-rod model to give the time evolution of long flexible fiber distribution with different linear densities. The fluctuation and velocity of fiber in jet flow were then studied numerically, and the simulation results can provide an optimization option for the air-jet loom to improve the energy efficiency by reasonably arranging the first assistant nozzle. The results are as follows.

(1) As the primary vortex rolls up, transports, and mixes with each other, the fiber fluctuation becomes stronger and the motion becomes more unstable as the linear density decreases.

(2) The fluctuation amplitude of a fiber with $\rho_{\mathrm{L}}=0.5 \times$ $10^{-5} \mathrm{~kg} \cdot \mathrm{m}^{-1}$ is two times larger than that of a fiber with $\rho_{\mathrm{L}}=2.0 \times 10^{-5} \mathrm{~kg} \cdot \mathrm{m}^{-1}$. It is as large as $3.34 \mathrm{~mm}$ when the fiber with $\rho_{\mathrm{L}}=0.5 \times 10^{-5} \mathrm{~kg} \cdot \mathrm{m}^{-1}$ arrives at $x_{\mathrm{A}}=120 \mathrm{~mm}$.

(3) The distance of the first assistant nozzle from the main nozzle should be less than $120 \mathrm{~mm}$ to avoid the collision between the fiber and the loom. With the increasing fiber linear density, the distance could be appropriately increased.

(4) The efficient length of the main nozzle to carry the fiber flying steadily forward is about $100-110 \mathrm{~mm}$. So an assistant nozzle should be added in this place.

(5) To save energy, according to (2) and (3), the suggested distance between the main nozzle and the first assistant nozzle is $110 \mathrm{~mm}$ for the fiber with $\rho_{\mathrm{L}}=$ $0.5 \times 10^{-5} \mathrm{~kg} \cdot \mathrm{m}^{-1}$. When the fiber linear density increases, the distance could be appropriately increased to $140 \mathrm{~mm}$.

\section{Conflicts of Interest}

The authors declare that they have no conflicts of interest.

\section{Acknowledgments}

This work was supported by the National Natural Science Foundation of China (Grant nos. 51576180 and 51676173), Fluid Engineering Innovation Team of Zhejiang Sci-Tech University (ZSTU) of China (Grant no. 11132932611309), National Scholarship Foundation of China, and 521 Talents Fostering Program Funding of Zhejiang Sci-Tech University of China.

\section{References}

[1] K. Mitugu, “Air jet loom,” US Patent 4,673,005, 1987.

[2] S. Yamamoto and T. Matsuoka, "A method for dynamic simulation of rigid and flexible fibers in a flow field," Journal of Chemical Physics, vol. 98, no. 1, pp. 644-650, 1993.

[3] S. De Meulemeester, J. Githaiga, L. Van Langenhove, D. V. Hung, and P. Puissant, "Simulation of the dynamic yarn behavior on air jet looms," Textile Research Journal, vol. 75, no. 10, pp. 724-730, 2005.

[4] S. B. Lindström and T. Uesaka, "Simulation of the motion of flexible fibers in viscous fluid flow," Physics of Fluids, vol. 19, no. 11, p. 113307, 2007.

[5] K. Vahidkhah and V. Abdollahi, "Numerical simulation of a flexible fiber deformation in a viscous flow by the immersed boundary-lattice Boltzmann method," Communications in Nonlinear Science \& Numerical Simulation, vol. 17, no. 3, pp. 1475-1484, 2012.

[6] K. K. Kabanemi and J. Hétu, "Effects of bending and torsion rigidity on deformation and breakage of flexible fibers: a direct simulation study," Journal of Chemical Physics, vol. 136, no. 7, p. 74903, 2012.

[7] G. Meirson and A. N. Hrymak, "Two-dimensional longflexible fiber simulation in simple shear flow," Polymer Composites, vol. 37, no. 8, pp. 2425-2433, 2015.

[8] W. Nan, Y. Wang, and H. Tang, "A viscoelastic model for flexible fibers with material damping," Powder Technology, vol. 276, pp. 175-182, 2015.

[9] S. D. Meulemeester, P. Puissant, and L. V. Langenhove, "Three-dimensional simulation of the dynamic yarn behavior on air-jet looms," Textile Research Journal, vol. 79, no. 18, pp. 1706-1714, 2009.

[10] S. D. Kim, J. I. Seo, and D. J. Song, “A computational analysis of unsteady transonic/supersonic flows over backward facing step in air jet nozzle," Journal of Mechanical Science \& Technology, vol. 21, no. 21, pp. 336-347, 2007.

[11] J. Andrić, S. T. Fredriksson, S. B. Lindström, S. Sasic, and H. Nilsson, "A study of a flexible fiber model and its behavior in DNS of turbulent channel flow," Acta Mechanica, vol. 224, no. 10, pp. 2359-2374, 2013.

[12] Y. Jin, J. Li, L. Zhu, J. Du, Y. Jin, and P. Lin, "Threedimensional numerical simulation of the movement of the flexible body under different constraints," Journal of Thermal Science, vol. 23, no. 6, pp. 593-599, 2014. 
[13] W. Yang, K. Zhou, Z. Zhao, and Z.-H. Wan, "Study on the two-way coupling turbulent model and rheological properties for fiber suspension in the contraction," Journal of NonNewtonian Fluid Mechanics, vol. 246, pp. 1-9, 2017.

[14] Z. Pei and C. Yu, "Numerical study on the effect of nozzle pressure and yarn delivery speed on the fiber motion in the nozzle of Murata vortex spinning," Journal of Fluids and Structures, vol. 27, no. 1, pp. 121-133, 2011.

[15] M. Ishida and A. Okajima, "Flow characteristics of the main nozzle in an air-jet loom part I: measuring flow in the main nozzle," Textile Research Journal, vol. 64, no. 1, pp. 10-20, 1994.

[16] D. D. Liu, Z. H. Feng, B. H. Tan, and Y. P. Tang, "Numerical simulation and analysis for the flow field of the main nozzle in an air-jet loom based on Fluent," Applied Mechanics and Materials, vol. 105, pp. 172-175, 2012.

[17] J. M. Lu, "Discussion on weft insertion processing parameters of air-jet loom," Applied Mechanics \& Materials, vol. 556-562, pp. 1005-1008, 2014.

[18] L. Chen, Z.-H. Feng, T.-Z. Dong, W.-H. Wang, and S. Liu, "Numerical simulation of the internal flow field of a new main nozzle in an air-jet loom based on Fluent," Textile Research Journal, vol. 85, no. 15, pp. 1590-1601, 2015.

[19] X. Liu and J. Lu, "Unsteady flow simulations in a three-lobe positive displacement blower," Chinese Journal of Mechanical Engineering, vol. 27, no. 3, pp. 575-583, 2014.

[20] J. Zhao, S. Zhou, X. Lu, and D. Gao, "Numerical simulation and experimental study of heat-fluid-solid coupling of double flapper-nozzle servo valve," Chinese Journal of Mechanical Engineering, vol. 28, no. 5, pp. 1030-1038, 2015.

[21] H. Sun, R. Xiao, F. Wang, Y. Xiao, and W. Liu, "Analysis of the pump-turbine s characteristics using the detached eddy simulation method," Chinese Journal of Mechanical Engineering, vol. 28, no. 1, pp. 115-122, 2014.

[22] J. Lin, X. Shi, and Z. Yu, "The motion of fibers in an evolving mixing layer," International Journal of Multiphase Flow, vol. 29, no. 8, pp. 1355-1372, 2003.

[23] L. Jianzhong, S. Xing, and Y. Zhenjiang, "Effects of the aspect ratio on the sedimentation of a fiber in Newtonian fluids," Journal of Aerosol Science, vol. 34, no. 7, pp. 909-921, 2003.

[24] J. H. Ferziger and M. Perić, Computational Methods for Fluid Dynamics, Springer, Berlin, Germany, 2002.

[25] H.-F. Guo, B.-G. Xu, C.-W. Yu, and S.-Y. Li, "Simulating the motion of a flexible fiber in 3D tangentially injected swirling airflow in a straight pipe-Effects of some parameters," International Journal of Heat and Mass Transfer, vol. 54, no. 21, pp. 4570-4579, 2011.

[26] W. Forstall and A. H. Shapiro, "Momentum and mass transfer in coaxial gas jets," Journal of Applied Mechanics-transactions of the ASME, vol. 17, pp. 399-408, 1950.

[27] P. Lin, D. Wu, M. Yu et al., "Large eddy simulation of the particle coagulation in high concentration particle-laden planar jet flow," Chinese Journal of Mechanical Engineering, vol. 24, no. 6, pp. 947-956, 2011.

[28] Y. Jin, Research on the Characteristics of Yarn-Air Flow Field Stimulated by Series Nozzles, Zhejiang University, Hangzhou, China, 2010. 


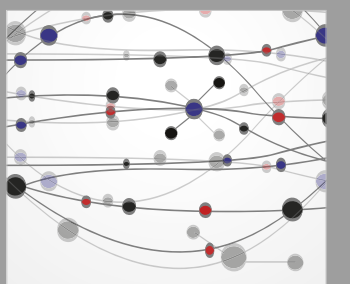

The Scientific World Journal
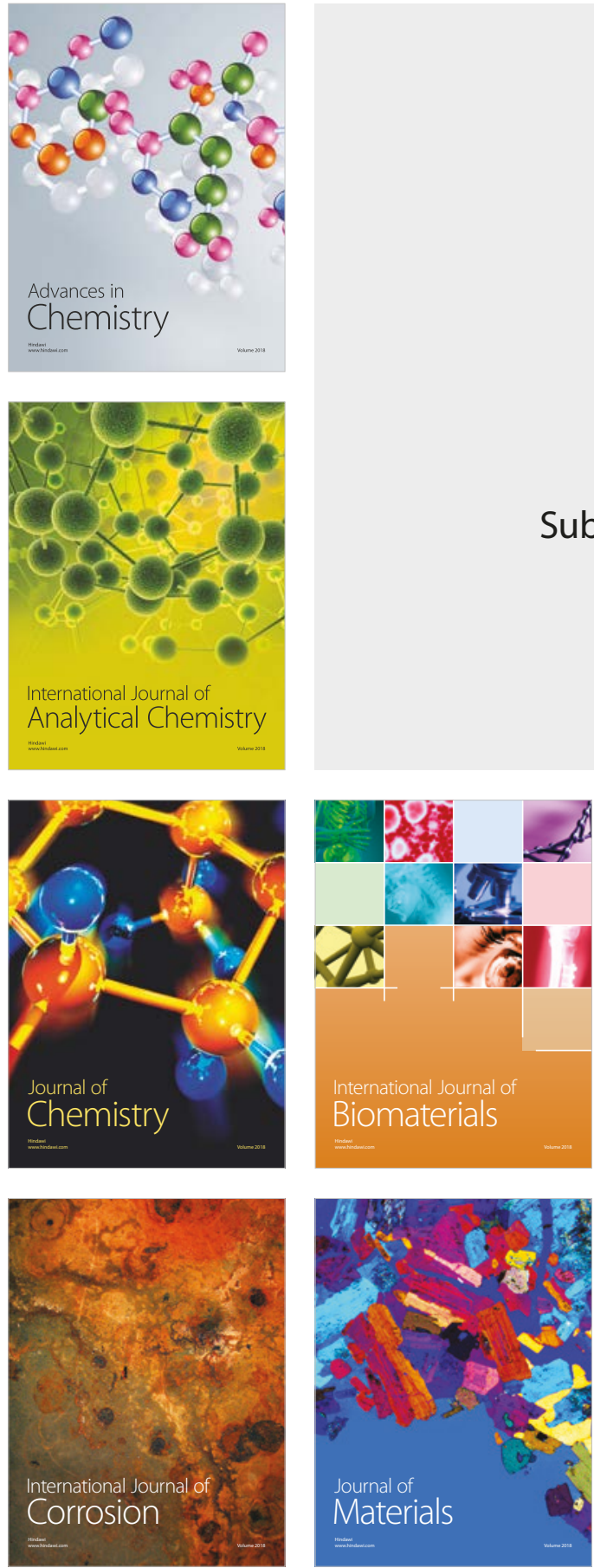

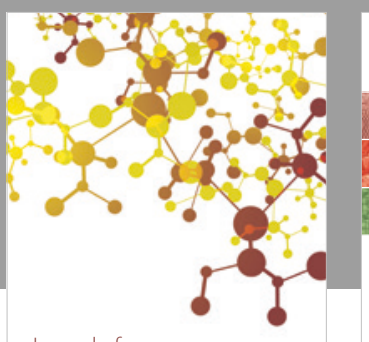

Journal of

Applied Chemistry
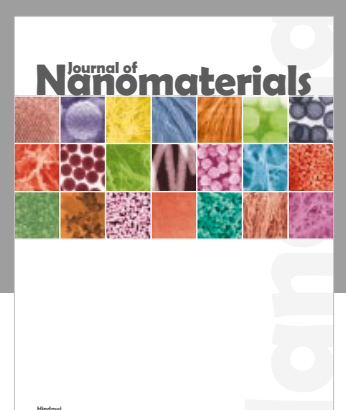

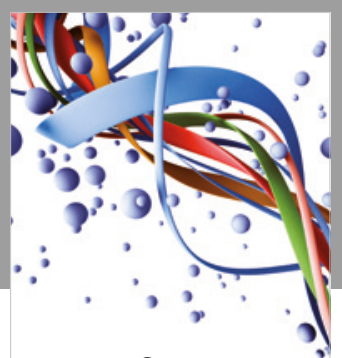

Scientifica

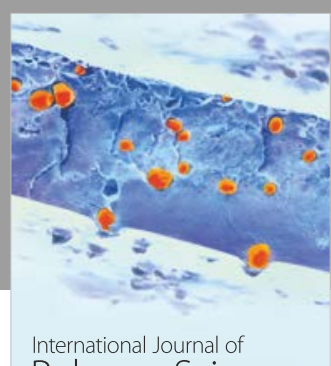

Polymer Science

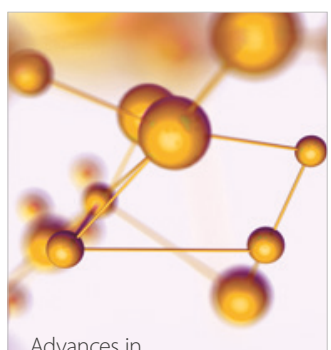

Physical Chemistry
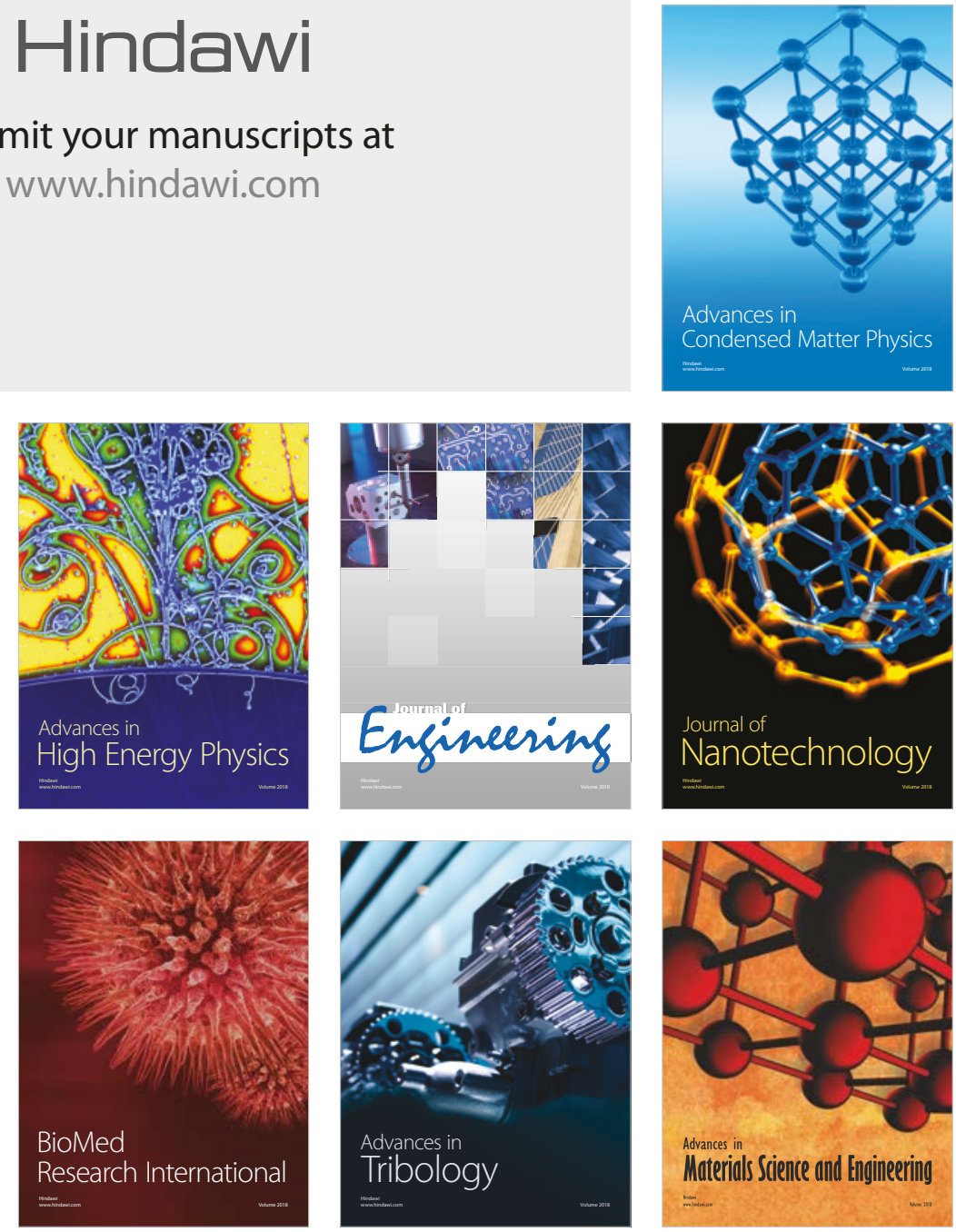